\title{
Forames mentuais anômalos: Relato de caso e considerações anatômicas
}

\author{
Anomalous mental foramina: Case report and anatomical considerations \\ Agujeros mentonianos anómalos: Reporte de caso y consideraciones anatómicas
}

Recebido: 26/03/2021 | Revisado: 31/03/2021 | Aceito: 03/04/2021 | Publicado: 13/04/2021

\author{
Yuri Barbosa Alves \\ ORCID: https://orcid.org/0000-0002-0036-1995 \\ Universidade Federal da Paraíba, Brasil \\ E-mail: ybarbosaa@gmail.com \\ Luiz Felipe Fernandes Gonçalves \\ ORCID: https://orcid.org/0000-0001-6847-7524 \\ Universidade Federal da Parába, Brasil \\ E-mail: 1felipefg@yahoo.com.br \\ Lucas Rodrigues Pinheiro \\ ORCID: https://orcid.org/0000-0002-4164-1838 \\ Centro Universitário do Pará, Brasil \\ E-mail: lucasrpinheiro@gmail.com \\ Marcelo Augusto Oliveira de Sales \\ ORCID: https://orcid.org/0000-0002-1575-6594 \\ Universidade Federal da Paraíba, Brasil \\ E-mail: marceloxray.sales@gmail.com
}

\begin{abstract}
Resumo
O forame mentual é uma importante estrutura anatômica que se apresenta geralmente por uma abertura única, bilateralmente na região vestibular do corpo da mandíbula, situado adjacente ao ápice dos pre-molares inferiores. Variações anatômicas podem ocorrer, como a presença de forames mentuais acessórios, que devem ser cuidadosamente avaliados no planejamento pré-operatório na região, afim de evitar danos aos feixes neurovasculares que emergem dos forames. O presente relato descreve a presença de forames mentuais acessórios bilateralmente na mandíbula, detectados através de tomografia computadorizada de feixe cônico prévio a cirurgia de colocação de implantes dentários. Dentro desse contexto, a avaliação tomográfica se faz mandatória, no intuito de ausência de danos ao feixe vásculo-nervoso e ocorrência de parestesias pós-operatórias.
\end{abstract}

Palavras-chave: Variação anatômica; Anatomia; Tomografia computadorizada de feixe cônico; Mandíbula.

\begin{abstract}
The mental foramen is an important anatomical structure, usually present as through a single (bilateral) opening in the vestibular portion of the mandible, located adjacent to the apex of the lower premolars. Anatomical variations occur, such as accessory mental foramina, which must be carefully evaluated during preoperative planning to avoid damage to their emergent neurovascular bundles. The present study describes the detection/presence of bilateral accessory mental foramen in the mandible via cone beam computed tomography prior to dental implant surgery.
\end{abstract}

Keywords: Anatomic Variation; Anatomy; Cone Beam computer Tomography; Mandible.

\section{Resumen}

El foramen mentoniano es una estructura anatómica importante que suele presentarse a través de una única abertura, bilateralmente en la región vestibular del cuerpo mandibular, ubicada adyacente al ápice de los premolares inferiores. Pueden ocurrir variaciones anatómicas, como la presencia de foramen mentoniano accesorio, que deben ser cuidadosamente evaluados en la planificación preoperatoria en la región, a fin de evitar daño a los haces neurovasculares que emergen de los foramen. El presente informe describe la presencia de foramen mentoniano accesorio bilateralmente en la mandíbula, detectado por tomografía computarizada de haz cónico previo a la cirugía de colocación de implantes dentales.

Palabras clave: Variación anatómica; Anatomía; Tomografía computarizada de haz cónico; Mandíbula.

\section{Introdução}

O forame mentual (FM) é uma importante estrutura anatômica que se apresenta geralmente por uma abertura única, oval ou circular, bilateralmente na região vestibular do corpo da mandíbula, localizado, adjacente ao ápice dos pre-molares inferiores. Através do FM que vasos sanguíneos e o nervo mentual emergem, responsáveis, respectivamente, pela vascularização 
e inervação da pele do mento, lábio, mucosa oral e gengiva (Iwanaga et al., 2016).

Variações anatômicas do FM vem sendo discutida na literatura, como diferenças em tamanho, forma, posição e número, tendo como destaque alterações relacionadas à presença de forames adicionais adjacente ao forame mentual, sendo denominado de forame mentual acessório (FMA). Existem poucos casos relatados na literatura de cinco forames em um mesmo indivíduo (Budhiraja et al., 2013; Katakami et al., 2008; Lauhr et al., 2015; Naitoh et al., 2009; Borghesi et al., 2018).

Os FMA possuem a mesma importância do FM, devendo ser, cuidadosamente avaliados no planejamento préoperatório, a fim de evitar distúrbios sensoriais pós-operatorio que podem levar a um resultado desfavorável no tratamento executado. Eles podem ser observados nas radiografias convencionais e nas tomografias computadorizadas de feixe cônico (TCFC), entretanto, por serem menores que $1,0 \mathrm{~mm}$, sua identificação se torna mais difícil através dos exames bidimensionais (Fuakami et al., 2011; Torres et al., 2015). Sendo a TCFC uma ferramenta de diagnóstico que fornece informações com maior acurácia sobre as estruturas do complexo maxilo-facial, possibilitando a identificação e avaliação de variações anatômicas, o relato deste estudo descreve a morfologia de forames mentuais atípicos, com um forame mentual em cada hemi-mandíbula, dois forames acessórios na hemi-mandíbula direita e um acessório na hemi-mandíbula esquerda, totalizando a presença de cinco forames.

\section{Metodologia}

O artigo se propõe a apresentar uma variação anatômica rara por meio de relato de caso com objetivos descritivos, exploratórios, com abordagem qualitativa (Pereira et al., 2018). Assim, propiciando o conhecimento desta condição anômala, enfatizando a importância do exame de imagem tomográfico em procedimentos cirúrgicos mais invasivos próximo a estruturas anatômicas importantes. Foram respeitados os princípios éticos da declaração de helsinque, além do paciente ter assinado e concordado com o termo de consentimento livre e esclarecido.

\section{Relato de Caso}

Paciente do sexo masculino, 63 anos, parcialmente edêntulo, foi submetido a TCFC (PreXion 3D, Prexion Inc., San Jose, CA, USA) no pré-operatório para o planejamento de cirurgia de implante, com protocolo de aquisição com voxel de 0.08 $\mathrm{mm}$. Após ajustes e reorientação das imagens nos três planos de orientação foi observado a presença de forames mentuais acessórios em cada hemi-mandíbula.

Na secção axial, observou-se a presença de dois forames na hemi-mandíbula do lado esquerdo, sendo um acessório e um principal (Figura 1) e três forames na hemi-mandíbula do lado direito, sendo dois acessórios e um principal (Figuras $2 \mathrm{~A}$ e 2B). Esses achados também foram visualizados no plano parassagital em direção distomesial, como observa-se na sequência de imagens (Figura 3). 
Research, Society and Development, v. 10, n. 4, e35510414294, 2021

(CC BY 4.0) | ISSN 2525-3409 | DOI: http://dx.doi.org/10.33448/rsd-v10i4.14294

Figura 1. Corte axial de hemi-mandíbula esquerda. MFleft: Forame mentual. FMA1: Forame acessório mentual.

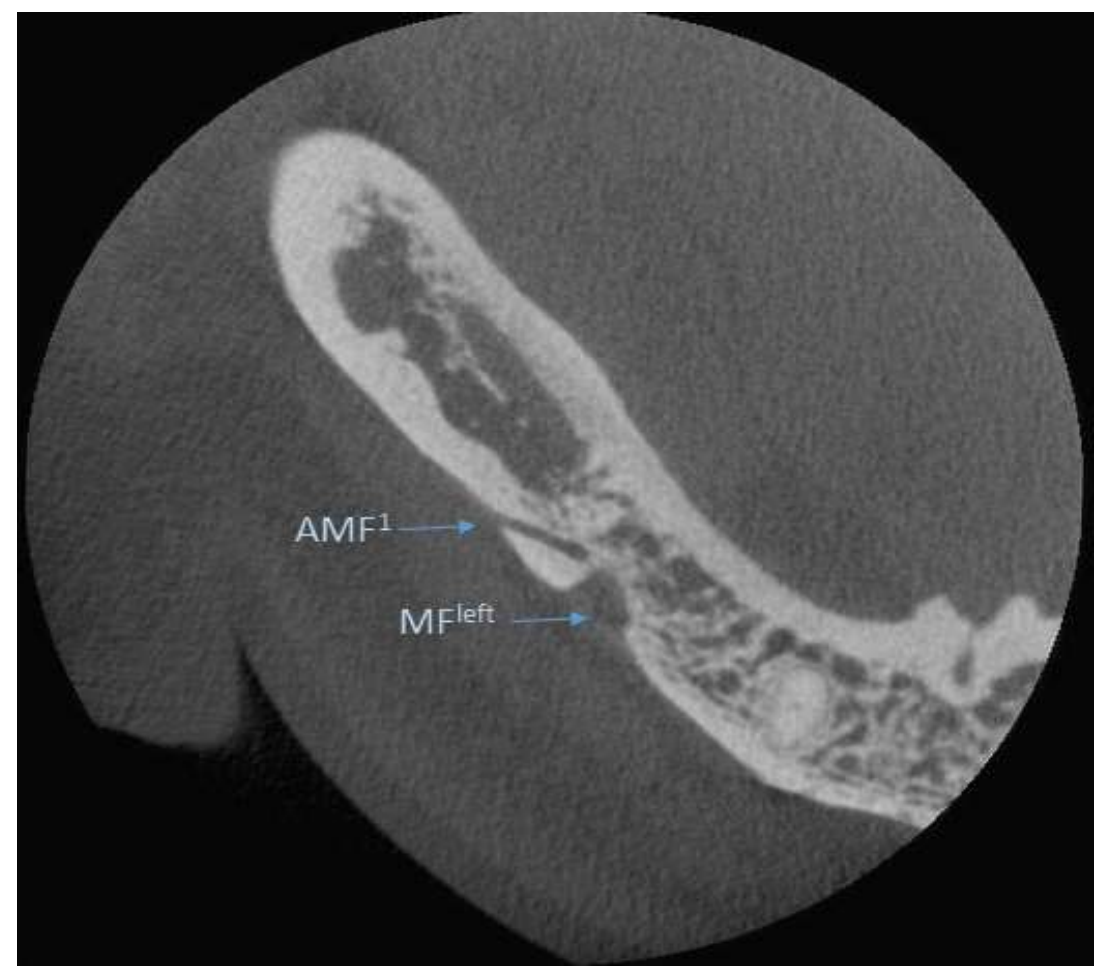

Fonte: Autores (2020)

Figura 2. Corte axial de hemi-mandíbula direita. (A) MFrigth: Forame mentual. AMF2: Forame mentual acessório. (B) AMF3: Forame mentual acessório.

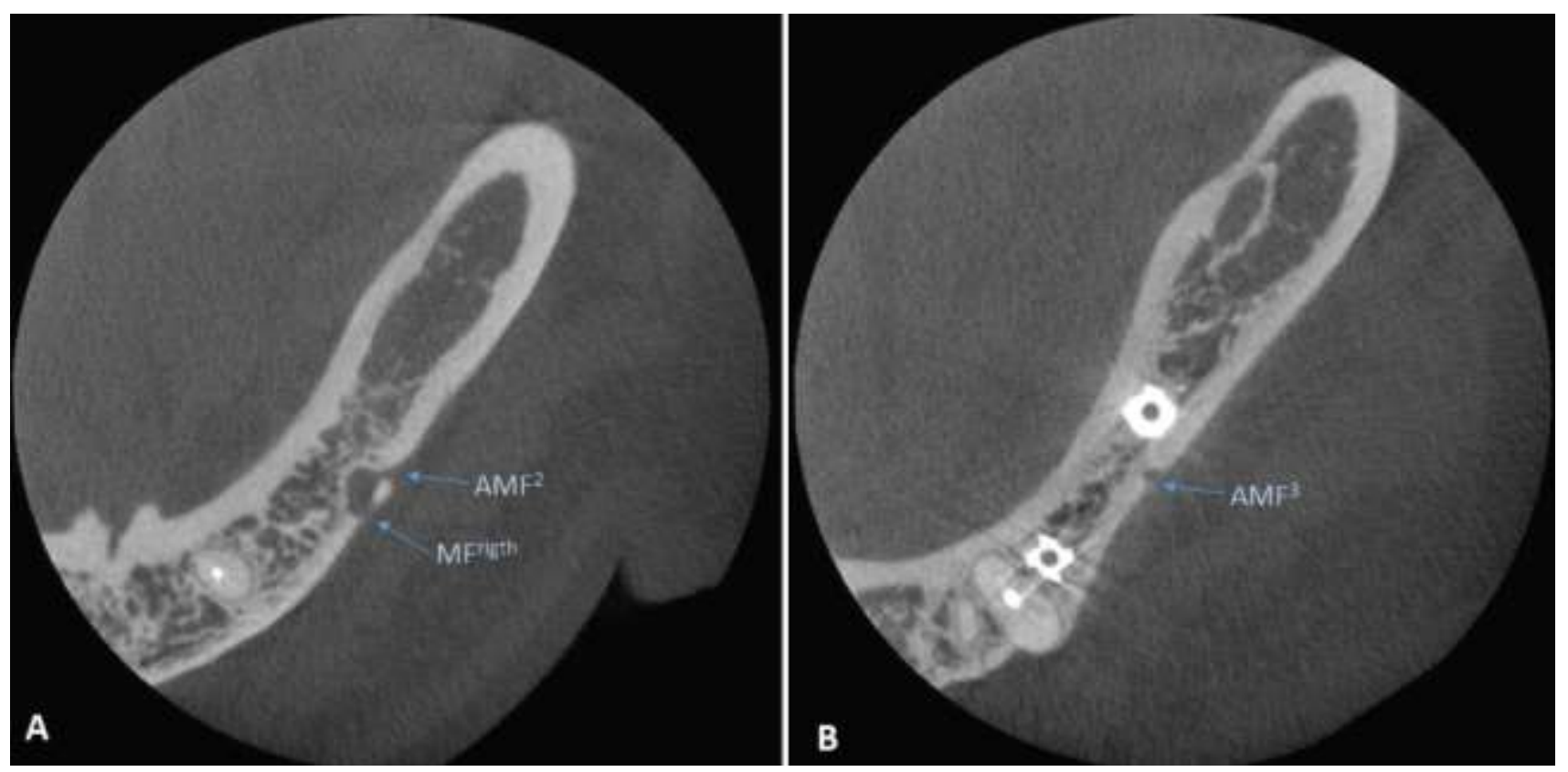

Fonte: Autores (2020) 
Figura 3. Sequência parassagital em direção distomesial. Setas indicam dois forames na hemi-manbíbula do lado esquerdo e três forames na hemi-mandíbula do lado direito.
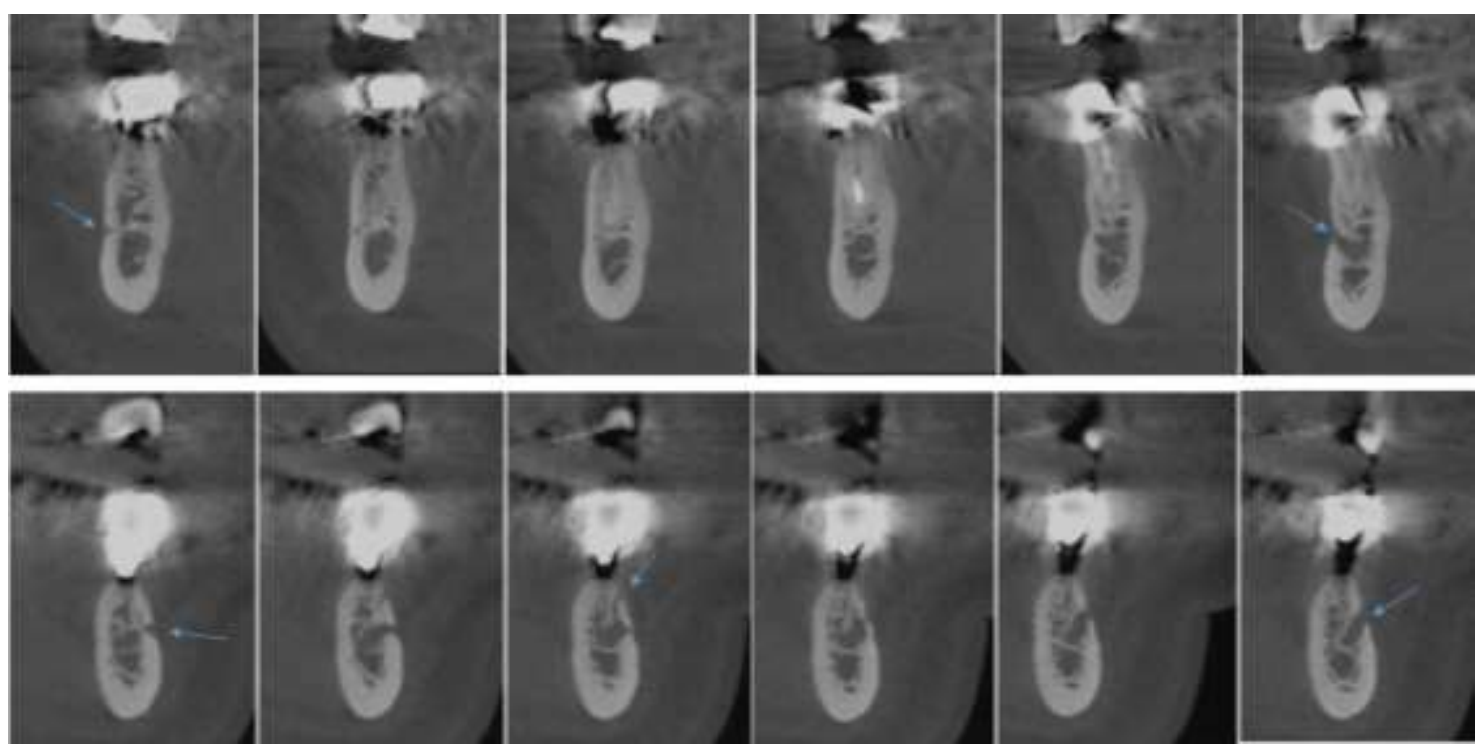

Fonte: Autores (2020)

Antes de sua abertura na porção vestibular, do lado esquerdo da mandíbula, o canal mandibular apresentou uma ramificação dirigindo-se para posterior com um curso intra-ósseo distinto e independente, caracterizando-se como um FMA no nível do segundo pré-molar. Tanto o FM como o FMA estavam localizados na mesma altura, sendo os diâmetros médios medindo, $3.35 \mathrm{~mm}$ e $0.88 \mathrm{~mm}$, respectivamente (Tabela 1).

No lado direito foi observado que o canal mandibular apresentava três aberturas, sendo uma principal e duas adicionais, em direção à cortical ósseo vestibular. Os três forames estavam localizados no nível entre os dois pré-molares, sendo o FM situado em sentido mesial e os forames mentuais acessórios em sentido distal. No plano parassagital observou-se que um FMA estava localizado em sentido apical, e o outro em sentido coronal. O diâmetro médio do FM e dos dois FMA foram $2.37 \mathrm{~mm}$, $1.21 \mathrm{~mm}$ e $1.05 \mathrm{~mm}$, respectivamente (Tabela 1).

Tabela 1. Diâmetro dos forames acessórios e mentuais na mandíbula. MF: Forame mentual. AMF: Forame mentual acessório.

\begin{tabular}{|c|c|c|c|c|}
\hline $\begin{array}{c}\text { Forame } \\
s\end{array}$ & $\begin{array}{c}\text { Orientaçã } \\
\boldsymbol{O}\end{array}$ & Diâmetro horizontal (mm) & $\begin{array}{c}\text { Diâmetro } \\
\text { vertical }(\mathrm{mm})\end{array}$ & $\begin{array}{c}\text { Diâmetro } \\
\text { médio }(\mathrm{mm})\end{array}$ \\
\hline MF $^{\text {left }}$ & Esquerdo & 3.22 & 3.49 & 3.35 \\
\hline $\mathrm{AMF}^{1}$ & Esquerdo & 0.89 & 0.87 & 0.88 \\
\hline $\mathrm{MF}^{\text {rigth }}$ & Direito & 2.57 & 2.17 & 2.37 \\
\hline $\mathrm{AMF}^{2}$ & Direito & 1.49 & 0.92 & 1.21 \\
\hline $\mathrm{AMF}^{3}$ & Direito & 1.01 & 1.08 & 1.05 \\
\hline
\end{tabular}

Fonte: Autores (2020)

A presença dos dois forames do lado esquerdo e dos três forames do lado direito da mandíbula foram demonstrados em reconstrução tridimensional (3D) (Figura 4). 
Figura 4. Reconstrução tridimensional (3D).

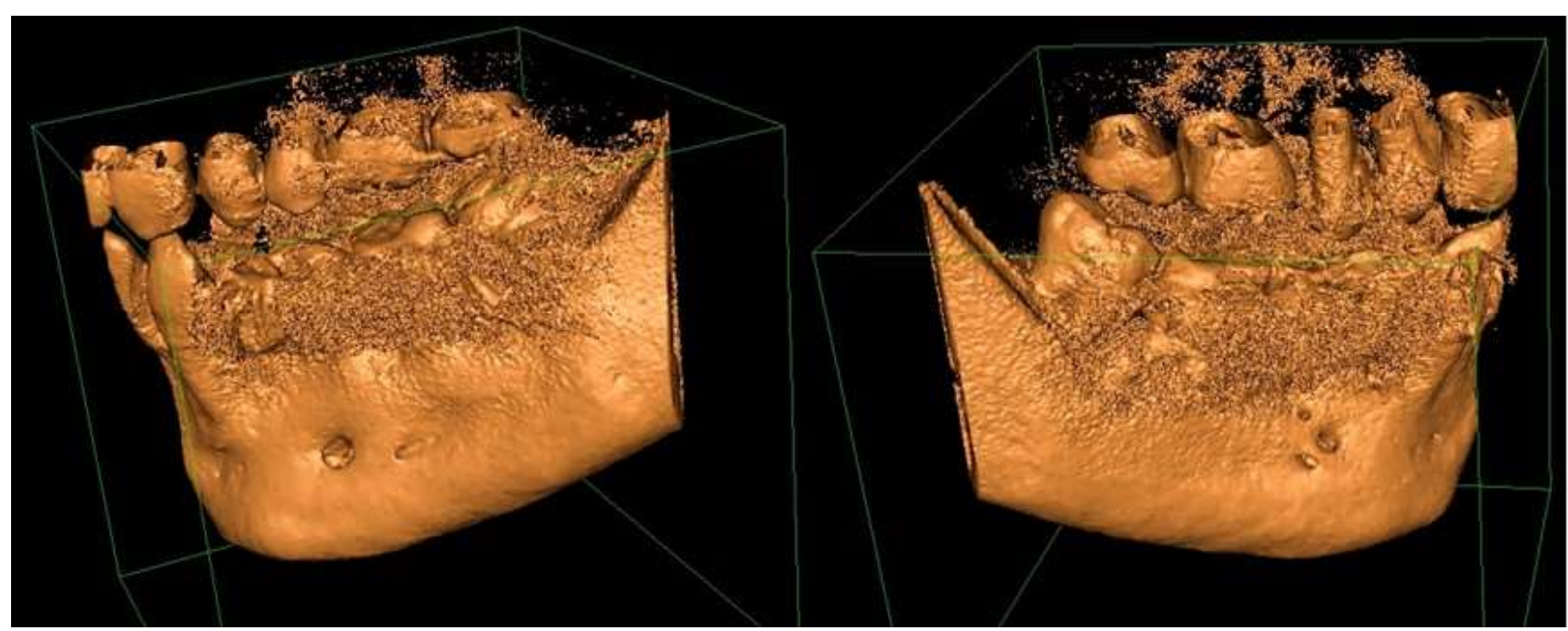

Fonte: Autores (2020)

\section{Discussão}

A identificação precisa do forame mentual e suas características antropométricas são importantes para o sucesso durante os procedimentos cirúrgicos realizados na mandíbula, como a colocação de implantes dentários e cirurgias ortognáticas. Sendo essencial a realização da TCFC previamente a uma cirurgia nessa região (Rahpeyma et al., 2018; Wei et al., 2020; Tyndal., 2012).

A presença e a observação de forames mentuais acessórios tornaram-se mais evidentes após a tomografia computadorizada, devido a dificuldade em sua visualização através das radiografias panorâmicas. Embora os métodos tradicionais de imagem bidimensional (2D) sejam frequentemente usados na odontologia, eles geralmente não têm sucesso no diagnóstico das variações anatômicas do FM (Imada et al., 2014). Em razão da alta resolução tridimensional da TCFC, este exame é particularmente adequado para avaliar detalhes anatômicos de forma refinada, sendo a ferramenta de diagnóstico de escolha no estudo de diferentes variações anatômicas do forame mentual. Em radiografias panorâmicas, a visualização do FMA só foi possível ser observada em 48,6\% dos casos visualizados em imagens de TCFC. Métodos de avaliação através de radiografia panorâmica são limitados, podendo subestimar a variação morfológica do MF, não sendo um exame confiável para avaliação no planejamento pré-cirúrgico (Goyushov et al., 2017).

Diversos relatos na literatura têm abordado as variações do forame mentual, seja relacionado com sua localização ou quantidade (Li et al., 2018; Lauhr et al., 2015). Normalmente a mandíbula humana apresenta apenas um único forame mentual em cada lado, no entanto a presença de forames adicionais tem sido descrita na literatura (Borghesi et al., 2018; Torres et al., 2015; Goyushov et al., 2017; Muinelo-Lorenzo et al., 2015; Vieira et al., 2018). Em estudo realizado com TCFC de 645 pacientes com idade média de 41 anos, 75 (11.6\%) foram diagnosticados com FMA, destes pacientes com forames mentuais acessórios, 67 (88\%) consistia de FMA unitário, 6 (10\%) FMA duplo e 2 (1.2\%) FMA triplo (Gumusok et al., 2017). E em estudo com 362 TCFC, foi encontrada prevalência de 9.4\% de FMA, sendo a maioria dos casos unilaterais, e apenas um caso com FMA duplo (Oliveira-Santos et al., 2011).

Foi observado na literatura uma variação de prevalência do FMA variando entre 1,9\% e 12,5\% em estudos radiológicos (Goyushov et al., 2017; Goyushov et al; 2018). O tipo de variação mais frequente é a presença de forame mentual duplo, que tem uma incidência relatada variando de 1,4 a 12,5\%, por sua vez, a incidência de forame mentual triplo é mais rara, variando 
de 0,7 a 1,2\% (Naitoh et al., 2011; Sawyer et al., 1998). Com relação ao sexo, a literatura tem reportado maior prevalência de FMA em homens do que em mulheres (Han et al., 2016). No presente estudo, foram encontrados dois forames na hemi-mandíbula esquerda, sendo que o mesmo paciente do sexo masculino também apresentou três forames do lado oposto, configurando uma excepcionalidade anatômica do FM.

Nos últimos anos, poucos artigos sobre múltiplos forames mentais foram publicados. Em um dos estudos foi relatado a variação anatômica do FM de um homem de 24 anos, dentado, que apresentou um FM, e dois FMA do lado direito, e de um FM e um FMA do lado esquerdo (Borghesi et al., 2018). Enquanto que Bejeh and Haghanifar descreveram, em um homem de 62 anos, edêntulo, a ocorrência de um FM e dois FMA do lado esquerdo, e de um FM e um FMA do lado direito, e mais um FMA pela face lingual na hemi-mandíbula direita. Estes dados sinalizam semelhança com o presente estudo em relação à presença de forames mentuais acessórios em indivíduos do sexo masculino (Bejer, Haghanifar, 2015).

Segundo estudo de Kalender et al., em que foi utilizado TCFC para determinar a ocorrência e localização de forames mentuais em 92 pacientes do sexo masculino, foi observado, no lado direito, diâmetro médio do FM igual a 3.85 mm, e do FMA $1.5 \mathrm{~mm}$, e para o lado esquerdo, essas medidas foram de $3.75 \mathrm{~mm}$ e $1.5 \mathrm{~mm}$, respectivamente. Como os valores para o FM foram maiores que do FMA da mesma hemi-mandíbula, esses dados estão em conformidade com o presente estudo, porém, diferentemente, as medidas lineares deste relato de caso foram menores (Kalender, Orhan, Aksoy, 2012).

No que diz respeito à forma dos forames mentuais, alguns estudos afirmaram que a oval seria a mais comum (Budhiraja et al., 2013; Naitoh et al., 2009), estado de acordo com este relato de caso, em que 3 dos 5 forames observados apresentaram essa morfologia.

A localização do FMA depende do FM e das raízes dos dentes posteriores (Gümüsok et al., 2017). De acordo com Bejeh and Haghanifar, em comparação com o FM, o diâmetro do FMA geralmente pode ser menor, e possuir uma localização para distal. Este dado corrobora com o presente relato de caso, em que tanto o FMA único do lado esquerdo e os dois FMA do lado direito, apresentaram um diâmetro mais reduzido, e em uma posição para distal, quando comparados com o FM. Enquanto que nos resultados encontrados por Iwanaga et al., o FMA pode ser maior e estar localizado de forma mesial em relação ao FM.

O estudo de imagem pré-operatório é importante antes de qualquer procedimento cirúrgico na região mentual (Direk et al., 2018). Em pacientes com inervação acessória, espera-se que ocorra falha anestésica em 10-20\% dos casos se apenas o nervo alveolar inferior for bloqueado (Boronat, Peñarrocha, 2006). Além disso, distúrbios neurossensoriais e hemorragias foram relatados após cirurgias nessa área. A disfunção sensorial devido ao dano do nervo na mandíbula pode ocorrer se o nervo alveolar inferior ou mentual for danificado durante a preparação de uma osteotomia (Greenstein, Tarnow, 2006). Portanto, atenção especial ao FMA e ao conteúdo vásculo-nervoso são importantes durante qualquer procedimento cirúrgico que envolva a região do FM, podendo reduzir a taxa de parestesia, paralisia e hemorragia na região mental.

\section{Considerações Finais}

O FM e suas potenciais variaç̃oes, como o FMA, são estruturas anatômicas neurovasculares importantes da mandíbula, e sua detecção e conhecimento precisos devem ser seriamente levados em consideração para procedimentos odontológicos cirúrgicos seguros e bem-sucedidos. Dentro desse contexto, a avaliação tomográfica se faz mandatória com objetivo de evitar danos aos feixes vásculos-nervosos e parestesias pós-operatórias. Sendo a TCFC o exame de imagem padrão ouro para avaliação pré-cirúrgica da região mandibular.

\section{Referências}

Bejeh Mir, A., \& Haghanifar, S. (2015). Accessory mental foramina, incisive nerve plexus and lingual canals with unusual emergence paths: Report of two rare cases. Indian J Dent, 6(1), 44-8. 
Borghesi, A., Pezzotti, S., Nocivelli, G., \& Maroldi, R. (2018). Five mental foramina in the same mandible: CBCT findings of an unusual anatomical variant. Surg Radiol Anat, 40(6), 635-40.

Boronat López, A., \& Peñarrocha Diago, M. (2006). Failure of locoregional anesthesia in dental practice. Review of the literature. Med Oral Patol Oral Cir Bucal, 11(6), 510-3.

Budhiraja, V., Rastogi, R., Lalwani, R., Goel, P., \& Bose, S. C. (2013). Study of Position, Shape, and Size of Mental Foramen Utilizing Various Parameters in Dry Adult Human Mandibles from North India. ISRN Anat, 1-5.

Direk, F., Uysal, I. I., Kivrak, A. S., Fazliogullari, Z., Unver Dogan, N., \& Karabulut, A. K. (2018). Mental foramen and lingual vascular canals of mandible on MDCT images: anatomical study and review of the literature. Anat Sci Int, 93(2), 244-53.

Fuakami, K., Shiozaki, K., Mishima, A., Shimoda, S., Hamada, Y., \& Kobayashi, K. (2011). Detection of buccal perimandibular neurovascularisation associated with accessory foramina using limited cone-beam computed tomography and gross anatomy. Surg Radiol Anat, 33(2), 141-6.

Goyushov, S., Tözüm, M. D., \& Tözüm, T. F. (2017). Accessory Mental/Buccal Foramina. Implant Dent, $26(5), 796-801$.

Goyushov, S., Tözüm, M. D., \& Tözüm, T. F. (2018). Assessment of morphological and anatomical characteristics of mental foramen using cone beam computed tomography. Surg Radiol Anat, 40(10), 1133-9.

Greenstein, G., \& Tarnow, D. (2006). The Mental Foramen and Nerve: Clinical and Anatomical Factors Related to Dental Implant Placement: A Literature Review. J Periodontol, 77(12), 1933-43.

Gümüsok, M., Akarslan, Z., Başman, A., \& Üçok, Ö. (2017). Evaluation of accessory mental foramina morphology with cone-beam computed tomography. Niger J Clin Pract, 20(12), 1550-4.

Han, S-S., Hwang, J. J., \& Jeong, H-G. (2016). Accessory mental foramina associated with neurovascular bundle in Korean population. Surg Radiol Anat, $38(10), 1169-74$.

Imada, T. S. N., Fernandes, L. M. P. da S. R., Centurion, B. S., de Oliveira-Santos, C., Honório, H. M., \& Rubira-Bullen, I. R. F. (2014). Accessory mental foramina: prevalence, position and diameter assessed by cone-beam computed tomography and digital panoramic radiographs. Clin Oral Implants Res, 25(2), 94-9

Iwanaga, J., Watanabe, K., Saga, T., Tabira, Y., Kitashima, S., Kusukawa, J., et al. (2016). Accessory mental foramina and nerves: Application to periodontal, periapical, and implant surgery. Clin Anat, 29(4), 493-501.

Kalender, A., Orhan, K., \& Aksoy, U. (2012). Evaluation of the mental foramen and accessory mental foramen in Turkish patients using cone-beam computed tomography images reconstructed from a volumetric rendering program. Clin Anat, 25(5), 584-92.

Katakami, K., Mishima, A., Shiozaki, K., Shimoda, S., Hamada, Y., \& Kobayashi, K. (2008). Characteristics of Accessory Mental Foramina Observed on Limited Cone-beam Computed Tomography Images. J Endod, 34(12), 1441-5.

Lauhr, G., Coutant, J. C., Normand, E., Laurenjoye, M., \& Ella, B. (2015). Bilateral absence of mental foramen in a living human subject. Surg Radiol Anat, $37(4), 403-5$.

Li, Y., Yang, X., Zhang, B., Wei, B., \& Gong, Y. (2018). Detection and characterization of the accessory mental foramen using cone-beam computed tomography. Acta Odontol Scand, 76(2), 77-85.

Muinelo-Lorenzo, J., Suárez-Quintanilla, J. A., Fernández-Alonso, A., Varela-Mallou, J., \& Suárez-Cunqueiro, M. M. (2015). Anatomical characteristics and visibility of mental foramen and accessory mental foramen: Panoramic radiography vs. cone beam CT. Med Oral Patol Oral Cir Bucal, $20(6), 707-14$.

Naitoh, M., Hiraiwa, Y., Aimiya, H., Gotoh, K., \& Ariji, E. (2009). Accessory mental foramen assessment using cone-beam computed tomography. Oral Surgery, Oral Med Oral Pathol Oral Radiol Endodontology, 107(2), 289-94.

Naitoh, M., Yoshida, K., Nakahara, K., Gotoh, K., \& Ariji, E. (2011). Demonstration of the accessory mental foramen using rotational panoramic radiography compared with cone-beam computed tomography. Clin Oral Implants Res, 22(12), 1415-9.

Oliveira-Santos, C., Souza, P. H. C., De Azambuja Berti-Couto, S., Stinkens, L., Moyaert, K., Van Assche, N., et al. (2011). Characterisation of additional mental foramina through cone beam computed tomography. J Oral Rehabil, 38(8), 595-600.

Pereira, A. S., et al. (2018). Metodologia da pesquisa científica. UFSM. https://repositorio.ufsm.br/bitstream/handle/1/15824/Lic_Computacao_MetodologiaPesquisa-Cientifica.pdf?sequence=1.

Rahpeyma, A., \& Khajehahmadi, S. (2018). Accessory Mental Foramen and Maxillofacial Surgery. J Craniofac Surg, 29(3), $216-7$.

Sawyer, D. R., Kiely, M. L., \& Pyle, M. A. (1998). The frequency of accessory mental foramina in four ethnic groups. Arch Oral Biol, 43(5), 417-20.

Torres, M. G. G., de Valverde, L. F., Andion Vidal, M. T., \& Crusoé-Rebello, I. M. (2015). Accessory mental foramen: A rare anatomical variation detected by cone-beam computed tomography. Imaging Sci Dent, 45(1), 61-5.

Tyndall, D. A., Price, J. B., Tetradis, S., Ganz, S. D., Hildebolt, C., \& Scarfe, W. C. (2012). Position statement of the American Academy of Oral and Maxillofacial Radiology on selection criteria for the use of radiology in dental implantology with emphasis on cone beam computed tomography. Oral Surg Oral Med Oral Pathol Oral Radiol, 113(6), 817-26.

Vieira, C. L., Veloso, S. do A. R., \& Lopes, F. F. (2018). Location of the course of the mandibular canal, anterior loop and accessory mental foramen through cone-beam computed tomography. Surg Radiol Anat, 40(12), 1411-7. 
Research, Society and Development, v. 10, n. 4, e35510414294, 2021

(CC BY 4.0) | ISSN 2525-3409 | DOI: http://dx.doi.org/10.33448/rsd-v10i4.14294

Wei, X., Gu, P., Hao, Y., \& Wang, J. (2020). Detection and characterization of anterior loop, accessory mental foramen, and lateral lingual foramen by using cone beam computed tomography. J Prosthet Dent, 124(3), 365-71.

WHO (World Health Organization). (1999). Proposed revision of the Declaration of Helsinki. Bulletin of Medical Ethics, 150, 18-22. 